\title{
Some Results on the Quantum Dynamics of a Particle in a Markovian Potential
}

\author{
Claude-Alain Pillet \\ Theoretical Physics, ETH-Hönggerberg, CH-8093 Zürich, Switzerland
}

\begin{abstract}
We consider the quantum dynamics of a particle in a time dependent potential $V(t)$, assuming it to be a Markovian random function of time. We derive a formula for the density matrix at time $t$ averaged over the realisations of the potential. We then obtain a kind of RAGE theorem for the time evolution of compact observables, and some information on the phase space behaviour of the system.
\end{abstract}

\section{Introduction and Results}

Since the pioneering work of Anderson [1] on localisation of electronic states in a static disordered lattice, random time independent Schrödinger operators have become increasingly popular in solid state physics to describe effects of impurities and thermal disorder in crystals. By now such models are also fairly well understood mathematically, see for example [2] and [3]. On the other hand time dependent random Hamiltonians received much less attention, probably because of the intrinsic difficulties encountered when dealing with non-autonomous systems. We mention some works (see for example [4]) on the $\delta(t)$-correlated gaussian random potential, typically a problem of the form:

$$
i \partial_{t} \psi_{t}=\left(-\Delta+V_{t}\right) \psi_{t} \text { on the lattice } \mathbb{Z}^{v}
$$

where $\Delta$ is the discrete Laplacian (or some tight binding Hamiltonian), and $V_{t}(x)$ a gaussian random field of mean zero and covariance

$$
\left\langle V_{t}(x) V_{s}(y)\right\rangle=g \delta(t-s) \delta_{x, y} .
$$

Such models can be explicitly solved to give a finite diffusion constant, i.e.

$$
0<\lim _{t \rightarrow \infty}\left\langle\frac{1}{t} \sum_{x} x^{2}\left|\psi_{t}(x)\right|^{2}\right\rangle=D<\infty,
$$

and thus a finite electrical conductivity. Another interesting special case is to take the potential $V_{t}$ in (1.1) as a Markovian random function of time. Such potentials can be constructed in the following way: choose a homogeneous Markov process $\xi(t)$ on 
some state space $E$ (for example $E$ a discrete set or a bounded region of $\mathbb{R}^{n}$ ) and a function $V(x, \xi)$, then simply set $V_{t}(x)=V(x, \xi(t))$. Such systems have been used to describe electron propagation in disordered media. However, the methods used are all approximate and difficult to control (see [16]). The purpose of this paper is to derive an exact formula for the mean time evolution of the density matrix associated with (1.1). It turns out that this evolution is rather simple, since it is given by a contraction semigroup, a fact reflecting the absence of memory of the potential. We also apply ergodic theory to this contraction semigroup to obtain information on the asymptotic behaviour of some observables, and then using a random ergodic theorem, we convert this mean result into an almost sure one.

In this paper we consider the continuum case, i.e. Equation (1.1) on $\mathbb{R}^{v}$, but it will be clear that all our results are also valid on the lattice with a bounded potential $V(x, \xi)$. In the continuum we assume $V(x, \xi)$ to be a small perturbation of $-\Delta$ uniformly in $\xi$, and a smooth function of $x$ and $\xi$. Furthermore we require $\xi(t)$ to be continuous or at least piecewise continuous (allowing for a discrete set of jumps); the precise hypotheses are exposed in Sect. 1.

Since our discussion is rather technical, we shall expose here the underlying heuristic ideas. Let $\rho\left(x, x^{\prime}\right)$ be a density matrix, its time evolution is governed by the equation,

$$
\partial_{t} \rho_{t}=-i\left[H(\xi(t)), \rho_{t}\right]=-K(\xi(t)) \rho_{t},
$$

where we have set $H(\xi)=-\Delta+V(\xi, x)$, and $K(\xi)=i[H(\xi), \cdot]$ a linear operator on the space of density matrices. If we allow for a dependence of the initial state $\rho_{0}$ of the system on the initial state of disorder $\xi(0)$, we are then interested in computing the expectation value

$$
E\left[\rho_{t}(\xi(0))\right]
$$

giving the mean state at time $t$. To do that we first look at the conditional expectation,

$$
E\left[\rho_{t}(\xi(0)) \mid \xi(t)=\xi\right]=\frac{E\left[\rho_{t}(\xi(0)) \delta(\xi(t)-\xi)\right]}{E[\delta(\xi(t)-\xi)]},
$$

from which (1.3) is obtained by integration over the (time independent) distribution of $\xi(t)$. To first order in $d t$ we have

$$
\rho_{t+d t}(\xi(0))=\rho_{t}(\xi(0))-K(\xi(t)) \rho_{t}(\xi(0)) d t
$$

So that taking expectation

$$
E\left[\rho_{t+d t}(\xi(0)) \mid \xi(t+d t)=\xi^{\prime}\right]=E\left[(1-K(\xi(t)) d t) \rho_{t}(\xi(0)) \mid \xi(t+d t)=\xi^{\prime}\right]
$$

Now using a well known property of conditional expectations and the Markov property

$$
E\left[(1-K(\xi(t)) d t) \rho_{t}(\xi(0)) \mid \xi(t), \xi(t+d t)\right]=E\left[(1-K(\xi(t)) d t) \rho_{t}(\xi(0)) \mid \xi(t)\right],
$$

since $\rho_{t}(\xi(0))$ is independent of $\xi(t+d t)$, we obtain

$$
E\left[\rho_{t+d t}(\xi(0)) \mid \xi(t+d t)=\xi^{\prime}\right]=E\left[E\left[(1-K(\xi(t)) d t) \rho_{t}(\xi(0)) \mid \xi(t)\right] \mid \xi(t+d t)=\xi^{\prime}\right] .
$$


If we now introduce the infinitesimal generator $A$ of the process $\xi(t)$ as

$$
\left(e^{-A(t-s)} f\right)(\xi)=E[f(\xi(s)) \mid \xi(t)=\xi] \quad(0<s<t),
$$

we can rewrite (1.4) to first order in $d t$ as

$$
\begin{aligned}
E\left[\rho_{t+d t}(\xi(0)) \mid \xi(t+d t)=\xi^{\prime}\right] & =\left(e^{-A d t} E\left[(1-K(\xi(t)) d t) \rho_{t}(\xi(0)) \mid \xi(t)=\cdot\right]\right)\left(\xi^{\prime}\right) \\
& =\left((1-(A+K) d t) E\left[\rho_{t}(\xi(0)) \mid \xi(t)=\cdot\right]\right)\left(\xi^{\prime}\right)
\end{aligned}
$$

where we have to interpret $K$ as a multiplication operator in $\xi$, i.e. $(K \rho)(\xi)=$ $K(\xi) \rho(\xi)$. The last identity can be recast in differential form as

$$
\partial_{t} E\left[\rho_{t}(\xi(0)) \mid \xi(t)=\cdot\right]=-(K+A) E\left[\rho_{t}(\xi(0)) \mid \xi_{t}=\cdot\right],
$$

and thus integrated to give

$$
E\left[\rho_{t}(\xi(0)) \mid \xi(t)=\xi\right]=\left(e^{-(K+A) t} \rho_{0}\right)(\xi),
$$

which is the promised semigroup formula. In Sect. 3 this is proven rigorously for a large class of equations of the type

$$
\frac{d}{d t} x(t)=-K(\xi(t)) x(t), \quad x(0)=x
$$

on abstract Banach spaces. This result is also of potential interest in other problems, like diffusion in disordered media, wave propagation etc....

The reader who accepts the above derivation may skip Sects. 2 and 3, going directly to the application given in Sect. 4. There we remark that under some very weak spectral assumption on the generator $(K+A)$, the mean ergodic theorem applied to the semigroup $e^{-(K+A) t}$ implies

$$
\lim _{T \rightarrow \infty} \frac{1}{T} \int_{0}^{T} E\left[\langle c\rangle_{\rho}(t)\right] d t=0
$$

for any compact observable $c$, where $\langle c\rangle_{\rho}(t)=\operatorname{tr}\left(c \rho_{t}\right)$ is the quantum mechanical expectation of $c$ in the state $\rho_{t}$. Our main achievement is then to remove the expectation symbol in formula (1.6) to obtain an almost sure result. This is done using a random ergodic theorem due to Beck and Schwartz [5]. At the end we discuss some consequences of this result on the phase space behaviour of the system.

Perhaps the most interesting consequence is for discrete systems (i.e. (1.1) on the lattice $\mathbb{Z}^{v}$ ). Then under the above assumptions we prove the absence of bound states in the following sense: in any state $\psi$ of the system, the mean occupation probability of any bounded region $B \subset \mathbb{Z}^{v}$ vanishes, i.e.

$$
\lim _{T \rightarrow \infty} \frac{1}{T} \int_{0}^{T} \sum_{x \in B}\left|\psi_{t}(x)\right|^{2} d t=0 .
$$

Finally let us note the analogy between our technique and the approach to time dependent (and in particular time periodic) Hamiltonians initiated by Howland in [20] and further developed by Yajima ([21]) and Yajima and Kitada ([22]). For a periodic Hamiltonian (of period 1) they introduce the augmented space

$$
L^{2}(\mathbb{R} / \mathbb{Z}, d t) \otimes \mathscr{H},
$$


where $\mathscr{H}$ is the usual quantum mechanical state space. On this space the self adjoint operator,

$$
P=-i \frac{\partial}{\partial t}+H(t)
$$

generates a unitary group which in some sense is equivalent to the quantum evolution generated by $H(t)$. In this case questions like existence of bound states, asymptotic completeness, etc..., may be answered in terms of spectral and scattering theory for $P$. This operator thus bears some analogy with the generator

$$
\mathscr{B}=[H(\xi), .]-i A
$$

of the mean evolution of density matrices in the Markovian case. There are however two major differences: first the semigroup generated by $\mathscr{B}$ only gives statistical information on the solutions to the time dependent Schrödinger equation (1.1) for a given class of potentials. More technical is the fact that, while $P$ is self adjoint, $\mathscr{B}$ is not, due to the dissipative effect of randomness (i.e. to the term $-i A$ ). At first sight this may seem troublesome, but the non-self adjoint (and perhaps non-spectral) operator $\mathscr{B}$ turns out to be much easier to analyse than $P$. The point is that for the large time behaviour of the corresponding semigroups only the real part of the spectrum is relevant. While for $P$ the nature of the spectrum (and hence the asymptotic behaviour of the state $\psi_{t}(x)$ ) depends very sensitively on the potential (with the possible occurrence of resonances, see for example [23] or [24]), it is very easy to control the eigenvalues of $\mathscr{B}$, the dissipative term $-i A$ always pushing them down in the lower half plane, thus making random systems much simpler than deterministic ones!

\section{Notation and Hypotheses}

a) The Driving Dynamics. We denote by $(\Xi, \mathbf{Z}, P)$ the underlying probability space: $\Xi$ is a set of paths $\xi=(\xi(t))_{t \geqq 0}$ on the topological state space $E, \mathbf{Z}$ a $\sigma$-algebra on $\Xi$ and $P$ a probability measure, we write $E[\cdot]$ for the associated expectation. We assume $P$-almost all paths in $\Xi$ to be right continuous and to have a finite number of discontinuities on any compact time interval. Introducing the past (respectively the future) $\sigma$-algebra $\mathbf{P}_{s}$ (respectively $\mathbf{F}_{s}$ ) at time $s$ as usual, the Markov property may be stated as:

$$
P[A \cap B \mid \xi(t)]=P[A \mid \xi(t)] P[B \mid \xi(t)] \quad \text { for any } A \in \mathbf{P}_{t} \quad \text { and } \quad B \in \mathbf{F}_{t} .
$$

Time translations are given by a family $\left(\tau_{t}\right)_{t \geqq 0}$ of measure preserving map, on $\Xi$ :

$$
\left(\tau_{t} \xi\right)(s)=\xi(t+s) \text {. }
$$

Finally $\mathbf{E}$ being the Borel $\sigma$-algebra on $E$ we assume the existence of a unique invariant measure $\mu$ on $(E, \mathbf{E})$ :

$$
P[A]=\int P[A \mid \xi(t)=\xi] d \mu(\xi) .
$$

We also introduce the Hilbert space

$$
\mathbf{h}=L^{2}(E, \mathbf{E}, d \mu)
$$


on which the operator family

$$
f \mapsto E[f(\xi(0)) \mid \underline{\xi}(t)=\xi] \quad t \geqq 0
$$

is well known to be a strongly continuous contraction semigroup. Let $A$ be its generator:

$$
\left(e^{-A t} f\right)(\xi)=E[f(\xi(0)) \mid \xi(t)=\xi] .
$$

Thus $A$ is a $m$-accretive operator on $\mathbf{h}$. If the process is symmetric, i.e. if:

$$
P[\xi(t) \in B \mid \xi(0)]=P[\xi(0) \in B \mid \xi(t)], \quad B \in \mathbf{E}, \quad t \geqq 0,
$$

then $A$ will be self-adjoint; however we do not assume that in the following.

b) The Evolution Equation. Now we describe the hypotheses on the generator of the evolution, essentially Kato's conditions [10] assuring existence and uniqueness of the solution to the initial value problem (1.5):

(i) The underlying space $X$ is a separable Banach space with the norm $\|\cdot\|$.

(ii) There is a second Banach space $Y$ with the norm $\|\cdot\|_{Y}$ densely and continuously embedded in $X$.

(iii) To each $\xi \in E$ there is a densely defined operator $K(\xi)$ on $X$ generating a strongly continuous contraction semigroup:

$$
e^{-K(\xi) t} \quad t \geqq 0
$$

(iv) $Y \subset \bigcap_{\xi \in E} D(K(\xi))$ so that $K(\xi) \in \mathbf{B}(Y, X)$. We also assume the map

$$
\xi \in E \mapsto K(\xi) \in \mathbf{B}(Y, X)
$$

to be norm continuous and bounded.

(v) There is an isometry $S: Y \rightarrow X$ such that

$$
S K(\xi) S^{-1}=K(\xi)+B(\xi) \quad B(\xi) \in \mathbf{B}(X)
$$

and $B(\cdot)$ is strongly continuous.

Then they are two constants $M$ and $\beta$ such that for $P$-a.a. paths $\xi$ a unique propagator

$$
U(\xi \mid t, s)=U(t, s) \quad 0 \leqq s \leqq t
$$

exists, with the properties:

(I) $\|U(t, s)\| \leqq 1, U(t, t)=I, U(t, s)=U(t, r) U(r, s)$.

(II) $U(t, s) Y \subset Y$ and $\|U(t, s)\|_{Y} \leqq M e^{\beta(t-s)}$.

(III) $U(t, s)$ is jointly strongly continuous in $(t, s)$ both in $X$ and $Y$.

(IV) For any $y \in Y$ and at each continuity point of the path $\xi$ :

$$
\frac{d}{d t} U(t, s) y=-K(\xi(t)) U(t, s) y \quad(X \text {-strongly }) .
$$

(V) For fixed $0 \leqq S<T$, let

$$
\xi_{(n)}(t)=\xi\left(S+j \frac{T-S}{n}\right) \text { for } S+j \frac{T-S}{n} \leqq t<S+(j+1) \frac{T-S}{n},
$$


then we have the bound

$$
\left\|\left\{U(\xi \mid T, S)-U\left(\xi_{(n)} \mid T, S\right)\right\} y\right\| \leqq M e^{\beta(T-S)} \int_{S}^{T}\left\|K(\xi(s))-K\left(\xi_{(n)}(s)\right)\right\|_{Y X} d s\|y\|_{Y}
$$

for any $y \in Y$, in particular,

$$
U(\xi \mid T, S)=s-\lim _{n \rightarrow \infty} U\left(\xi_{(n)} \mid T, S\right)=S-\lim _{n \rightarrow \infty} \prod_{j=0}^{n-1} e^{-K(\xi(S+j(T-S) / n))(T-S) / n} .
$$

(VI) $\|\{U(\xi \mid t, 0)-I\} y\| \leqq M e^{\beta t} \int_{0}^{t}\|K(\xi(s))\|_{Y X} d s\|y\|_{Y}$ for any $y \in Y$.

(VII) $U(\xi \mid t, s) x(\xi)$ is strongly measurable for all strongly measurable $X$-valued functions $x(\cdot)$.

The propositions (I)-(VI) are the results of Kato, thus we only give a Proof of (VII). First we note that for any $y \in Y$,

$$
\frac{d}{d s} e^{-K(\xi)(t-s)} e^{-K(\eta) s} y=e^{-K(\xi)(t-s)}\{K(\xi)-K(\eta)\} e^{-K(\eta) s} y,
$$

so that by integration

$$
\left\{e^{-K(\xi) t}-e^{-K(\eta) t}\right\} y=-\int_{0}^{t} e^{-K(\xi)(t-s)}\{K(\xi)-K(\eta)\} e^{-K(\eta) s} y d s,
$$

and using the bound (II) for the path $\xi(t) \equiv \eta$,

$$
\left\|e^{-K(\xi) t}-e^{-K(\eta) t}\right\|_{Y X}<t M e^{\beta t}\|K(\xi)-K(\eta)\|_{Y X},
$$

so we obtain the bound

$$
\begin{aligned}
& \left\|\left\{\prod_{j=1}^{n} e^{-K\left(\xi_{j}\right) t_{j}}-\prod_{j=1}^{n} e^{-K\left(\eta_{j}\right) t_{j}}\right\} y\right\| \\
& =\left\|\sum_{k=1}^{n} \prod_{j=1}^{k-1} e^{-K\left(\xi_{j}\right) t_{j}}\left\{e^{-K\left(\xi_{k}\right) t_{k}}-e^{-K\left(\eta_{k}\right) t_{k}}\right\} \prod_{j^{\prime}=k+1}^{n} e^{-K\left(\eta_{j^{\prime}}\right) t_{j^{\prime}}} y\right\| \\
& \quad \leqq \sum_{k=1}^{n} M e^{\beta} \sum_{j=k+1}^{n} t_{j} t_{k} M e^{\beta t_{k}}\left\|K\left(\xi_{k}\right)-K\left(\eta_{k}\right)\right\|_{Y X}\|y\|_{Y},
\end{aligned}
$$

where we have used (II) again. Thus by assumption (iv),

$$
\prod_{j=1}^{n} e^{-K\left(\xi_{j}\right) t_{j}} y
$$

is jointly continuous in $\xi_{1}, \xi_{2}, \ldots, \xi_{n}$, and so is Borel measurable. But since $U(\xi \mid t, 0) y$ is by (V) a norm limit of such expressions, it is also Borel measurable. Now any strongly measurable $X$-valued function $x(\cdot)$ on $E$ is by definition and (ii) a norm limit of $Y$-valued simple functions $y(\cdot)$ for which $U(\xi \mid t, 0) y(\xi)$ is Borel measurable, so that since the $U(\xi \mid t, 0)$ all are contractions, $U(\xi \mid t, 0) x(\xi)$ is Borel as norm limit of Borel functions. Finally since we assumed $X$ to be separable, strong measurability follows.

Note that as a by-product

$$
\prod_{j=1}^{n} e^{-K\left(\xi_{j}\right) t_{j}} x\left(\xi_{1}\right)
$$


is strongly measurable in $\left(\xi_{1}, \xi_{2}, \ldots, \xi_{n}\right) \in E^{n}$ for any strongly measurable $X$-valued function $x(\cdot)$ on $E$.

Remarks. (1) Separability of $X$ was only assumed to simplify measurability questions. In the general case Petti's theorem should do the job.

(2) Condition (iii) is not so restrictive as we may think, for if $K(\cdot)$ is stable in the sense of Kato [10], i.e.

$$
\left\|\prod_{j=1}^{n} e^{-K\left(\xi_{j}\right) t_{j}}\right\|<M_{0} e^{\beta_{0}} \sum_{j=1}^{n} t_{j}
$$

for some $M_{0}, \beta_{0}$, we may first absorb $\beta_{0}$ in $K$ and then introduce a new but equivalent norm on $X$ :

thus obtaining condition (iii).

$$
\|x\|^{\prime}=\sup _{\substack{n>0 \\ t_{1}, \ldots, t_{n} \geq 0 \\ \xi_{1}, \ldots, \xi_{n} \in E}}\left\|\prod_{j=1}^{n} e^{-K\left(\xi_{j}\right) t_{j}} x\right\|,
$$

Before ending this section we want to prove a Banach space version of the Markov property which will be useful in the next section:

Lemma 2.1. Assume the map $\Xi \rightarrow \mathbf{B}(X), \xi \mapsto B(\xi)$, is such that:

(i) $b=\underset{\xi \in \Xi}{\operatorname{essup}}\|B(\xi)\|<\infty$

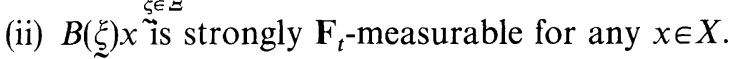

Then for any strongly $\mathbf{P}_{t}$-measurable, Bochner integrable $x(\cdot)$ :

$$
E[B(\xi) x(\xi) \mid \xi(t)]=E[B(\xi) E[x(\xi) \mid \xi(t)] \mid \xi(t)] \quad \mu \text { a.s. . }
$$

Proof. Assume first $x(\xi)=f(\xi) x$ with $x \in X$ and $f$ a $\mathbf{P}_{t}$ measurable essentially bounded function, then

$$
B(\xi) x(\xi)=f(\xi) B(\xi) x
$$

is strongly measurable, and since

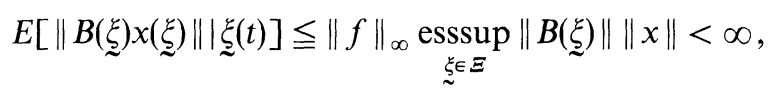

is also Bochner integrable. Then for any $l \in X^{*}$ a well known property of Bochner integrals and the ordinary Markov property give

$$
\begin{aligned}
\langle l, E[B(\xi) x f(\xi) \mid \xi(t)]\rangle & =E[\langle l, B(\xi) x\rangle f(\xi) \mid \xi(t)] \\
& =E[\langle l, B(\xi) x\rangle E[f(\xi) \mid \xi(t)] \mid \xi(t)] \\
& =\langle l, E[B(\xi) E[x(\xi) \mid \xi(t)] \mid \xi(t)]\rangle .
\end{aligned}
$$

Thus the desired identity holds in that case. But by definition of Bochner integrability (see for example [6]) there is a sequence $x_{n}(\cdot)$ of finite linear combinations of such functions such that

$$
\lim _{n \rightarrow \infty} x_{n}(\xi)=x(\xi) P \text {-a.s. and } \lim _{n \rightarrow \infty} E\left[\left\|x(\xi)-x_{n}(\xi)\right\|\right]=0 .
$$


Then the simple estimates

$$
\begin{gathered}
\left\|E[B(\xi) x(\xi) \mid \xi(t)]-E\left[B(\xi) x_{n}(\xi) \mid \xi(t)\right]\right\| \leqq b E\left[\left\|x(\xi)-x_{n}(\xi)\right\| \mid \xi(t)\right] \\
\left\|E[B(\xi) E[x(\xi) \mid \xi(t)] \mid \xi(t)]-E\left[B(\xi) E\left[x_{n}(\xi) \mid \xi(t)\right] \mid \xi(t)\right]\right\| \leqq b E\left[\left\|x(\xi)-x_{n}(\xi)\right\| \mid \xi(t)\right],
\end{gathered}
$$

combined with the triangle inequality give

$$
E[\|E[B(\xi) x(\xi) \mid \xi(t)]-E[B(\xi) E[x(\xi) \mid \xi(t)] \mid \xi(t)]\|] \leqq 2 b E\left[\left\|x(\xi)-x_{n}(\xi)\right\|\right],
$$

which using (2.2) give the desired result as we let $n \rightarrow \infty$.

\section{The Mean Evolution}

In this section we show that the mean solution to Eq. (1.5) is given by a contraction semigroup on the Banach space

$$
\mathbf{X}=L^{2}(E, \mathbf{E}, d \mu ; X),
$$

which is defined as the set of all $X$-valued strongly measurable functions on $E$ being norm square integrable,

$$
\|x\|_{\mathbf{X}}^{2}=\int\|x(\xi)\|^{2} d \mu(\xi)<\infty .
$$

The space $\mathbf{Y}$ is defined in the obvious analogous way. From assumption (ii) it follows that $\mathbf{Y}$ is densely and continuously embedded in $\mathbf{X}$. In the following we will drop the subscript $\mathbf{X}$ on the norm since no confusion can arise.

On $\mathbf{X}$ we define the operator family

$$
\left(Q^{t} x\right)(\xi)=E[U(\xi \mid t, 0) x(\xi(0)) \mid \xi(t)=\xi] .
$$

This makes sense by (VII) and the bound:

$$
\begin{aligned}
& \left\|Q^{t} x\right\|^{2}=\int \| E\left[U(\underset{\xi}{\xi} \mid t, 0) x(\underset{\Sigma}{\xi}(0)) \mid \xi(t)=\eta \|^{2} d \mu(\eta)\right. \\
& \leqq \int E\left[\|U(\xi \mid t, 0) x(\xi(0))\|^{2} \mid \xi(t)=\eta\right] d \mu(\eta) \\
& \leqq E\left[\|x(\xi(0))\|^{2}\right]=\|x\|^{2} \text {. }
\end{aligned}
$$

Thus $Q^{t}$ is a contraction on $\mathbf{X}$. In fact we now prove the

Lemma 3.1. $Q^{t}$ is a strongly continuous contraction semigroup on $\mathbf{X}$.

Proof. We first show the semigroup property:

$$
\begin{aligned}
& \left(Q^{t+s} x\right)(\xi)=E[U(\xi \mid t+s, 0) x(\underset{z}{\xi}(0)) \mid \xi(t+s)=\xi] \\
& =E[E[U(\xi \mid t+s, t) U(\xi \mid t, 0) x(\xi(0)) \mid \xi(t), \underset{z}{\xi}(t+s)=\xi] \mid \xi(t+s)=\xi] .
\end{aligned}
$$

Now by (V), (VII), $U(\xi \mid t, 0) x(\xi(0))$ is strongly $\mathbf{P}_{t}$-measurable and for any $x \in X, U(\xi \mid t+s, t) x$ is strongly $\mathbf{F}_{t}$-measurable, so application of Lemma 2.1 gives:

$$
\begin{aligned}
\left(Q^{t+s} x\right)(\xi) & =E[E[U(\xi \mid t+s, t) E[U(\xi \mid t, 0) x(\xi(0)) \mid \xi(t)] \mid \xi(t), \xi(t+s)=\xi] \mid \xi(t+s)=\xi] \\
& =E\left[U(\xi \mid t+s, t)\left\{Q^{t} x\right\}(\xi(t)) \mid \xi(t+s)=\xi\right] .
\end{aligned}
$$

By homogeneity and the fact that

$$
U(\xi \mid t+s, t)=U\left(\tau_{t} \xi \mid s, 0\right),
$$


we obtain

$$
\left(Q^{t+s} x\right)(\xi)=E\left[U(\xi \mid s, 0)\left\{Q^{t} x\right\}(\xi(0)) \mid \xi(s)=\xi\right]=\left(Q^{s} Q^{t} x\right)(\xi) .
$$

Now we prove strong continuity. Since $Q^{t}$ is a contraction, it suffices to prove it on a total subset of $\mathbf{X}$. We choose

$$
x(\xi)=f(\xi) y \quad \text { with } \quad f \in \mathbf{h} \text { and } y \in Y,
$$

then

$$
\begin{aligned}
\left\|\left(Q^{t}-I\right) x\right\|^{2}= & \int\|E[U(\xi \mid t, 0) y f(\xi(0))-y f(\xi(t)) \mid \xi(t)=\xi]\|^{2} d \mu(\xi) \\
\leqq & 2 \int\left(\|E[\{U(\xi \mid t, 0)-I\} y f(\xi(0)) \mid \xi(t)=\xi]\|^{2}\right. \\
& \left.+\|y\|^{2}\left|\left\{e^{-A t}-I\right\} f(\xi)\right|^{2}\right) d \mu(\xi) \\
\leqq & 2\left(E\left[\|\{U(\xi \mid t, 0)-I\} y\|^{2}|f(\xi(0))|^{2}\right]+\|y\|^{2}\left\|\left\{e^{-A t}-I\right\} f\right\|^{2}\right) \\
\leqq & 2\left(M^{2} e^{2 \beta t} t^{2} \sup _{\xi \in E}\|K(\xi)\|_{Y X}^{2}\|y\|_{Y}^{2}\|f\|^{2}+\|y\|^{2}\left\|\left\{e^{-A t}-I\right\} f\right\|^{2}\right),
\end{aligned}
$$

where we have used the bound (VI). Since the last expression clearly converges to zero as $t \downarrow 0$, the proof is complete.

To get a precise description of the generator of $Q^{t}$ we will need two further operators on $\mathbf{X}$ which we now define. By the remark in the proof of Lemma 2.1, the operator

$$
x(\xi) \mapsto e^{-K(\xi) t} x(\xi)
$$

makes sense on $\mathbf{X}$. That it is a contraction semigroup is clear. Strong continuity is an easy consequence of the bounds used in the proof of Lemma 2.1 and the dominated convergence theorem. We denote by $\mathbf{K}$ the generator of this semigroup:

$$
\left(e^{-\mathbf{K} t} x\right)(\xi)=e^{-K(\xi) t} x(\xi) \quad \mu \text {-a.s.. }
$$

Using the same technique, we can easily show $\mathbf{Y} \subset D(\mathbf{K})$ and

$$
(\mathbf{K} y)(\xi)=K(\xi) y(\xi) \quad \text { for } \quad y \in \mathbf{Y} \text {. }
$$

Further since $\mathbf{Y}$ is invariant under the semigroup, it is a core of $\mathbf{K}$.

The second operator we will need can be defined by

$$
x(\xi) \rightarrow E[x(\xi(0)) \mid \xi(t)=\xi] .
$$

By the proof of Lemma 3.1 (with $K(\xi) \equiv 0$ ), this is also a strongly continuous contraction semigroup on $\mathbf{X}$, the generator of which we call $\mathbf{A}$. The dense set $D(A) \otimes X$ (i.e. the set of finite linear combinations of terms of the form $f(\xi) x, f \in D(A)$ and $x \in X)$ is invariant and thus a core of A. Clearly: $(\mathbf{A} x f)(\xi)=x(A f)(\xi) \mu$-a.e.

Remarks. (3) Let $P^{t}(\xi \mid d \eta)=P[\xi(0) \in d \eta \mid \xi(t)=\xi]$ be the transition kernel of the process. Then

$$
\left(e^{-\mathbf{A} t} x\right)(\xi)=\int x(\eta) P^{t}(\xi \mid d \eta)
$$

(4) $D(A) \otimes Y$ is dense in $\mathbf{X}$ and contained in $D(\mathbf{A}) \cap D(\mathbf{K})$, so $\mathbf{B}_{0}=\mathbf{A}+\mathbf{K}$ is a densely defined accretive operator on $\mathbf{X}$.

We are now ready to characterise the generator of $Q^{t}$. 
Theorem 3.2. Let $\mathbf{B}_{0}=\mathbf{A}+\mathbf{K}$ on $D(\mathbf{A}) \cap D(\mathbf{K})$, and $Q^{t}=e^{-t \mathbf{B}}$, then $\mathbf{B}_{0} \subset \mathbf{B}$. In particular if $\operatorname{Ran}\left(\mathbf{B}_{0}+\lambda\right)$ is dense in $\mathbf{X}$ for some $\lambda>0$, then $\overline{\mathbf{B}}_{0}=\mathbf{B}$.

Proof: We fix $T>0$ and define $\xi_{(n)}(t)$ for $0 \leqq t \leqq T$ as in (V) (with $S=0$ ). Pick $x \in X$ and set

$$
\begin{gathered}
y_{n}(\xi)=U\left(\xi_{(n)} \mid t, 0\right) x(\underset{\xi}{\xi}(0)), \\
y(\xi)=U(\underline{\xi} \mid t, 0) x(\xi(0)) .
\end{gathered}
$$

By (V), $\lim _{n \rightarrow \infty} y_{n}(\xi)=y(\xi) P$-a.s., and since $\|y(\xi)\|$ and $\left\|y_{n}(\xi)\right\|$ all are bounded by $\|x(\xi)\|$, we have by the dominated convergence theorem:

$$
\lim _{n \rightarrow \infty} E\left[\left\|y(\xi)-y_{n}(\xi)\right\|^{2}\right]=0
$$

But then, setting

$$
\begin{aligned}
\left(Q_{n}^{t} x\right)(\xi) & =E\left[y_{n}(\xi) \mid \xi(t)=\xi\right], \\
\left\|\left\{Q^{t}-Q_{n}^{t}\right\} x\right\|^{2} & =\int\left\|E\left[y(\xi)-y_{n}(\xi) \mid \xi(t)=\xi\right]\right\|^{2} d \mu(\xi) \\
& \leqq \int E\left[\left\|y(\xi)-y_{n}(\xi)\right\|^{2} \mid \xi(t)=\xi\right] d \mu(\xi)=E\left[\left\|y(\xi)-y_{n}(\xi)\right\|^{2}\right] .
\end{aligned}
$$

So we have shown

$$
\operatorname{sim}_{n \rightarrow \infty} Q_{n}^{t}=Q^{t}
$$

We now seek an explicit formula for $Q_{n}^{t}$ :

i.e.

$$
\begin{aligned}
\left(Q_{n}^{t} x\right)(\xi) & =E\left[\prod_{j=0}^{n-1} e^{-K(\xi(j t / n)) t / n} x(\xi(0)) \mid \xi(t)=\xi\right] \\
& =\int \prod_{j=0}^{n-1} e^{-K\left(\xi_{j}\right) t / n} x\left(\xi_{0}\right) P^{t / n}\left(\xi \mid d \xi_{n-1}\right) P^{t / n}\left(\xi_{n-1} \mid d \xi_{n-2}\right) \cdots P^{t / n}\left(\xi_{1} \mid d \xi_{0}\right) \\
& =\int P^{t / n}\left(\xi \mid d \xi_{n-1}\right) e^{-K\left(\xi_{n-1}\right) t / n} \ldots P^{t / n}\left(\xi_{1} \mid d \xi_{0}\right) e^{-K\left(\xi_{0}\right) t / n} x\left(\xi_{0}\right) \\
& =\left(e^{-\mathbf{A} t / n} e^{-\mathbf{K} t / n} \cdots e^{-\mathbf{A} t / n} e^{-\mathbf{K} t / n} x\right)(\xi),
\end{aligned}
$$

$$
Q_{n}^{t}=\left(e^{-\mathbf{A} t / n} e^{-\mathbf{K} t / n}\right)^{n} .
$$

The result then follows by standard arguments on the Trotter product formula (see for example Davies [7]).

Thus defining $P: \mathbf{X} \rightarrow X$ by $P x=\int x(\xi) d \mu(\xi)$, we formulate our result as

$$
E[x(t)]=P e^{-\mathbf{B} t} x
$$

for the solution $x(t)$ to $(1.5)$.

\section{An Application to Quantum Mechanics}

We now consider the quantum mechanical system with time dependent Hamilton operator

$$
H(\xi(t))=-\Delta+V(\xi(t), x)
$$


$\Delta$ being the ordinary Laplacian on $\mathbf{H}=L^{2}\left(R^{v}\right)$, and $V$ a measurable function on $E \times R^{v}$. Assuming for some $a<1$ and a.a. $\xi \in E$,

$$
\left.\begin{array}{l}
D(V(\xi, \cdot)) \supset D(\Delta) \\
\forall \phi \in D(\Delta): \quad\|V(\xi, \cdot) \phi\| \leqq a\|\Delta \phi\|+b\|\phi\|
\end{array}\right\}
$$

formula (4.1) defines, via the Kato-Rellich theorem ([8]), a family of self-adjoint operators with common domain $D(H(\xi))=D(\Delta)$. Further assuming

$$
\left.\begin{array}{l}
\text { the function } V(\xi, \cdot)(1-\Delta)^{-1} \text { to the } \\
\text { bounded operators on } \mathbf{H} \text { to be norm continuous }
\end{array}\right\}
$$

we fulfill condition (iv) with $Y=D(\Delta)$. Finally choosing $S=1-\Delta$ and requiring

$$
\left.\begin{array}{l}
\sum_{k=1}^{v}\left[\frac{\partial^{2} V(\xi, x)}{\partial x_{k}^{2}}+2 \frac{\partial V(\xi, x)}{\partial x_{k}} \frac{\partial}{\partial x_{k}}\right](1-\Delta)^{-1} \\
\text { to be bounded and strongly continuous }
\end{array}\right\}(\mathrm{C}),
$$

we also assure condition $(v)$. Thus the unitary propagator $U(\xi \mid t, s)$ of the system satisfies (I)-(VII). Let us denote by $\mathscr{U}(\xi \mid t, s)$ the induced propagator of density matrices, and by $\mathscr{U}^{+}(\xi \mid t, s)$, its dual which propagates bounded observables,

$$
\mathscr{U}^{+}(\xi \mid t, s) C=U^{*}(\xi \mid t, s) C U(\xi \mid t, s) .
$$

We first prove an almost sure result on the propagation of compact observables:

Theorem 4.1. Let $\mathbf{C}$ be the space of compact operators on $\mathbf{H}$, then the ergodic mean

$$
\bar{C}(\xi)=\lim _{t \rightarrow \infty} \frac{1}{t} \int_{0}^{t} \mathscr{U}^{+}(\xi \mid s, o) C d s
$$

exists $P$-a.s. in the uniform topology and is a continuous linear map from $\mathbf{C}$ to $L^{1}(\Xi, d P ; \mathbf{C})$.

Proof. Using the density of finite rank operators in $\mathbf{C}$, it is not hard to see that the restriction of $\mathscr{U}^{+}(\xi \mid t, s)$ to compact operators is strongly continuous in $s$ and $t$, and strongly measurable in $\xi$, so the ergodic mean

$$
\frac{1}{t} \int_{0}^{t} \mathscr{U}^{+}(\xi \mid s, 0) C d s
$$

exists as a strong Riemann integral and is strongly measurable. Now set $t=n+\varepsilon$ with $0 \leqq \varepsilon<1$. Then (4.2) can be written as

$$
(n+\varepsilon)^{-1} \int_{n}^{n+\varepsilon} \mathscr{U}^{+}(\xi \mid s, 0) C d s+\frac{n}{n+\varepsilon} \frac{1}{n} \sum_{k=0}^{n-1} \int_{k}^{k+1} \mathscr{U}^{+}(\xi \mid s, 0) C d s,
$$

which as $n \rightarrow \infty$ is the same as

$$
\frac{1}{n_{k}} \sum_{=0}^{n-1} \int_{0}^{1} \mathscr{U}^{+}(\xi \mid 1,0) \ldots \mathscr{U}^{+}(\xi \mid k, k-1) \mathscr{U}^{+}(\xi \mid k+s, k) C d s .
$$

Using the time translation by one $\tau=\tau_{1}$ this becomes 


$$
\frac{1}{n_{k}} \sum_{=0}^{n-1} \mathscr{U}^{+}(\xi \mid 1,0) \ldots \mathscr{U}^{+}\left(\tau^{k-1} \xi \mid 1,0\right) \int_{0}^{1} \mathscr{U}^{+}\left(\tau^{k} \xi \mid s, 0\right) C d s .
$$

If we assume now $C$ to be Hilbert-Schmidt we are exactly in the situation of the random ergodic theorem of [5] which assures the $P$-a.s. existence of the uniform limit of (4.3) as $n \rightarrow \infty$ (we must first restrict $C$, because this theorem only works on reflexive Banach spaces). But then using the density of Hilbert-Schmidt operators in $\mathbf{C}$, it is easy to show that the limit exists for any compact operator. The asserted linearity and continuity is also an easy matter.

To get more information about the ergodic mean $\bar{C}(\xi)$ we now proceed to apply the result of Sect. 3 to $\mathscr{U}(\xi \mid t, 0)$. Using the well known isomorphism between Hilbert-Schmidt operators and $\mathbf{H} \otimes \mathbf{H}$, we may identify density matrices (which are trace class and hence Hilbert-Schmidt) with elements of $\mathbf{H} \otimes \mathbf{H}$. Doing so, the evolution equation for $\mathscr{U}(\xi \mid t, 0)$ becomes

$$
\frac{d}{d t} \mathscr{U}(\xi \mid t, 0)=-i\{H(\xi(t)) \otimes I-I \otimes H(\xi(t))\} \mathscr{U}(\xi \mid t, 0) .
$$

Thus setting $K(\xi)=i\{H(\xi) \otimes I-I \otimes H(\xi)\}$ and formally $\mathbf{B}=\mathbf{K}+\mathbf{A}$, we have

$$
E[\mathscr{U}(\xi \mid t, 0) \rho \mid \xi(t)=\xi]=\left(e^{-\mathbf{B} t} \rho\right)(\xi) .
$$

Let $C$ be some positive Hilbert-Schmidt operator and denote by $\langle$, $\rangle$ the inner product of $L^{2}(E, d \mu ; \mathbf{H} \otimes \mathbf{H})$. Then

$$
\begin{aligned}
\left\langle C, e^{-\mathbf{B} t} \rho\right\rangle & =\int \operatorname{tr}\left(C\left(e^{-\mathbf{B} t} \rho\right)(\xi)\right) d \mu(\xi)=E[\operatorname{tr}(C \mathscr{U}(\xi \mid t, 0) \rho)] \\
& =\operatorname{tr}\left(E\left[\mathscr{U}^{+}(\xi \mid t, 0) C\right] \rho\right) .
\end{aligned}
$$

Taking the ergodic mean of the last identity, we obtain

$$
\frac{1}{t} \int_{0}^{1}\left\langle C, e^{-\mathbf{B} s} \rho\right\rangle d s=\operatorname{tr}\left(E\left[\frac{1}{t} \int_{0}^{t} \mathscr{U}^{+}(\xi \mid s, 0) C d s\right] \rho\right) .
$$

If we now make the crucial assumption,

$\left(\sum\right)$ : zero is not an eigenvalue of $\mathbf{B}$,

(see the appendix for a simple criterion), we obtain as $t \rightarrow \infty$ in (4.4), using the mean ergodic theorem for contraction semigroups,

$$
0=\operatorname{tr}(E[\bar{C}(\xi)] \rho)=E[\operatorname{tr}(\bar{C}(\xi) \rho)] .
$$

Since $\rho$ is arbitrary and $C$ positive we easily see that $\bar{C}(\xi)=0 P$-a.s., and since any Hilbert-Schmidt operator is a finite linear combination of positive operators of the same class, the result extends to arbitrary Hilbert-Schmidt's and by continuity to all compact operators. Furthermore,

$$
\begin{aligned}
\frac{1}{t} \int_{0}^{t}\|C U(\xi \mid s, 0) f\|^{2} d s & =\frac{1}{t} \int_{0}^{t}\left(f, U(\xi \mid s, 0)^{*} C^{*} C U(\xi \mid s, 0) f\right) d s \\
& \leqq\|f\|^{2}\left\|\frac{1}{t} \int_{0}^{t} U(\xi \mid s, 0)^{*} C^{*} C U(\xi \mid s, 0) d s\right\| .
\end{aligned}
$$


Upon setting $F_{C}(\xi \mid t)=\left\|\frac{1}{t} \int_{0}^{t} U(\xi \mid s, 0)^{*} C^{*} C U(\xi \mid s, 0) d s\right\|$, we have proved the

Theorem 4.2 Under assumptions (A), (B), (C) and ( $\sum$ )

$$
\lim _{t \rightarrow \infty}\left\|\frac{1}{t} \int_{0}^{t} U(\xi \mid s, 0)^{*} C U(\xi \mid s, 0) d s\right\|=0 P \text {-a.s. }
$$

for any compact operator $C$ on $\mathbf{H}$. In particular there is a function $F_{C}(\xi \mid t)$ with

and

$$
\lim _{t \rightarrow \infty} F_{C}(\xi \mid t)=0 \quad P \text {-a.s. }
$$

$$
\frac{1}{t} \int_{0}^{t}\|C U(\xi \mid s, 0) f\|^{2} d s \leqq F_{C}(\xi \mid t)\|f\|^{2} .
$$

In the case of time independent potential, this RAGE theorem holds on the continuous spectral subspace of the Hamiltonian (see e.g. [9]), so we would expect our result to imply absence of bound states in some sense. However the lack of energy conservation introduces new complications, for the energy can now grow without bounds as $t \rightarrow \infty$. As already remarked by Enss and Veselić [11], time boundedness of energy is a very difficult question which, to our knowledge, has only been answered in explicitly solvable cases (see [11] for further comments and examples). No attempt will be made here to solve this problem.

A geometric characterisation of bound states was first proposed by Ruelle [12] on the basis of ergodic theory for unitary groups (see also [13] for further developments). It reads

$$
\mathbf{H}_{b}(\xi)=\mathbf{H}_{b}=\left\{f \in \mathbf{H} \mid \lim _{\boldsymbol{R} \rightarrow \infty} \sup _{t \geqq 0}\|F(|x|>R) U(\xi \mid t, 0) f\|=0\right\} .
$$

Further we may define a subspace of states having time bounded energy in a completely analogous way:

$$
\mathbf{H}_{\mathrm{be}}(\xi)=\mathbf{H}_{\mathrm{be}}=\left\{f \in \mathbf{H} \mid \lim _{E \rightarrow \infty} \sup _{t \geqq 0}\left\|F\left(H_{0}>E\right) U(\xi \mid t, 0) f\right\|=0\right\} .
$$

These two subspaces are closed, as is

$$
\mathbf{H}_{p}=\mathbf{H}_{b} \cap \mathbf{H}_{\mathrm{be}},
$$

which can also be characterised as the set of states with precompact trajectories

$$
\mathbf{H}_{p}=\{f \in \mathbf{H} \mid\{\overline{U(\xi \mid t, 0) f \mid t \geqq 0}\} \text { is compact in } \mathbf{H}\}
$$

(see [11] for an easy proof). We can now state the

Corollary 4.3. Under the assumptions of Theorem $4.2, \mathbf{H}_{p}=\{0\}$, i.e. they are no bound states of bounded energy.

In particular

$$
\lim _{T \rightarrow \infty} \frac{1}{T} \int_{0}^{T}\|F(|x|<R) U(\xi \mid t, 0) f\|^{2} d t=0 \quad \forall f \in \mathbf{H}_{\mathrm{be}}(\xi) .
$$


Proof. Let $f \in \mathbf{H}_{b} \cap \mathbf{H}_{\mathrm{be}}$. Then

$$
\begin{aligned}
\|f\|^{2}= & \frac{1}{T} \int_{0}^{T}\|U(\xi \mid t, 0) f\|^{2} d t \\
= & \frac{1}{T} \int_{0}^{T}\left\{\|F(|x|>R) U(\xi \mid t, 0) f\|^{2}+2\left\|F(|x|<R) F\left(H_{0}>E\right) U(\xi \mid t, 0) f\right\|^{2}\right. \\
& \left.+2\left\|F(|x|<R) F\left(H_{0}<E\right) U(\xi \mid t, 0) f\right\|^{2}\right\} d t \\
\leqq & \sup _{t \geqq 0}\|F(|x|>R) U(\xi \mid t, 0) f\|^{2}+2 \sup _{t \geqq 0}\left\|F\left(H_{0}>E\right) U(\xi \mid t, 0) f\right\|^{2} \\
+ & 2 \frac{1}{T} \int_{0}^{T}\left\|F(|x|<R) F\left(H_{0}<E\right) U(\xi \mid t, 0) f\right\|^{2} d t .
\end{aligned}
$$

First allowing $T \rightarrow \infty$ and using Theorem 4.4:

$$
\|f\|^{2} \leqq \sup _{t \geqq 0}\|F(|x|>R) U(\xi \mid t, 0) f\|^{2}+2 \sup _{t \geqq 0}\left\|F\left(H_{0}>E\right) U(\xi \mid t, 0) f\right\|^{2},
$$

since $F(|x|<R) F\left(H_{0}<E\right)$ is compact. Letting then $R, E \rightarrow \infty$, we obtain $f=0$. The last statement of the corollary is proven in the same way.

Remark (5). We worked in the continuum $\mathbb{R}^{v}$, but clearly all our results also hold on the lattice $\mathbb{Z}^{v}$ with a tight-binding Hamiltonian $H(t)=-\Delta+V(\xi(t), x)$. Then the energy is always bounded, so there are no bound states in this case,

$$
\mathbf{H}_{b}=\{0\} .
$$

Finally let us make the further assumption:

$$
\text { (D) }\left\{\begin{array}{l}
|V(\xi, x)| \leqq C(1+|x|)^{-(2+\varepsilon)} \\
\text { for some } C, \varepsilon \geqq 0 .
\end{array}\right.
$$

Then by Cook's method (see [9]) the wave operator

$$
\Omega(\xi)=s-\lim _{t \rightarrow+\infty} U(\xi \mid t, 0)^{*} e^{i \Delta t}
$$

exists. The vectors in the range of $\Omega(\xi)$ are called scattering states, $\mathbf{H}_{s}=\operatorname{Ran} \Omega(\xi)$, because they have an asymptotically free motion:

$$
\phi \in \mathbf{H}_{s} \Rightarrow \lim _{t \rightarrow+\infty}\left\|U(\xi \mid t, 0) \phi-e^{-i \Delta t} \psi\right\|=0 \quad \text { for some } \psi \in \mathbf{H} .
$$

It then clearly follows that $\mathbf{H}_{s} \subset \mathbf{H}_{\text {be }}$. We have the following

Corollary 4.4. If $v \geqq 5$, then $\mathbf{H}_{s}=\mathbf{H}_{\mathrm{be}}$.

Proof. Jafaev proved (see [17]):

$$
\mathbf{H}_{s}(\xi)=\left\{f \in \mathbf{H} \mid \forall R>0 \exists\left\{t_{n}\right\}, t_{n} \rightarrow \infty,\left\|F(|x|<R) U\left(\xi \mid t_{n}, 0\right) f\right\| \rightarrow 0 \text { as } n \rightarrow \infty\right\},
$$

but by Corollary 4.3 any $f \in \mathbf{H}_{\text {be }}$ has the desired property.

Remarks. (6) In some cases it is possible to show the wave operator $\Omega$ to be unitary. Then $\mathbf{H}=\mathbf{H}_{s}=\mathbf{H}_{\mathrm{be}}$. This occurs for example: 
(i) for short range repulsive potential (see [18]),

(ii) in the weak coupling case (see [19]).

(7) More elaborated consequences of our formalism will be published in a forthcoming paper.

\section{Appendix: The Condition $(\Sigma)$}

We begin with a simple

Lemma. Assume $\mathscr{A}$ to be $m$-sectorial (see [14]) and $u \in D(\mathscr{B})$ with $\mathscr{B} u=0$. Then $u \in D(\mathscr{A}) \cap D(\mathscr{K})$ and $\mathscr{A} u=\mathscr{K} u=0$.

Proof. Since $\mathscr{B}$ is accretive the quadratic form

$$
q(f)=\operatorname{Re}(f, \mathscr{B} f) \quad \text { on } D(q)=D(\mathscr{B})
$$

is closable with closure $\bar{q}$. Restricting this form to $D(A) \otimes H^{2}\left(R^{2 v}\right)$ which is a core of $\mathscr{A}$, we obtain the form

$$
q_{0}(f)=\operatorname{Re}(f,(\mathscr{A}+\mathscr{K}) f)=\operatorname{Re}(f, \mathscr{A} f),
$$

since $\mathscr{K}$ is anti self-adjoint. But $\mathscr{A}$ is $m$-sectorial, so it has the representation

$$
\mathscr{A}=\mathscr{C}(I+i \mathscr{D}) \mathscr{C} \quad \text { with } \quad \mathscr{C}=\mathscr{C}^{*} \geqq 0 \quad \text { and } \quad\|\mathbf{D}\|<1, \quad D(\mathbf{C})=Q(\mathscr{A}) \supset D(\mathscr{A}) \text {. }
$$

Thus

$$
q_{0}(f)=\|\mathscr{C} f\|^{2}, \quad q_{0} \subset q \subset \bar{q} .
$$

Clearly $q_{0}$ is also closable with closure

$$
\bar{q}_{0}(f)=\|\mathscr{C} f\|^{2}, \quad D\left(\bar{q}_{0}\right)=D(\mathscr{C})=Q(\mathscr{A}) \quad \text { and } \quad \bar{q}_{0} \subset \bar{q} .
$$

But since $\mathscr{C}$ is self-adjoint the form $\bar{q}_{0}$ has no proper closed extension, i.e.

So

$$
\bar{q}=\bar{q}_{0}
$$

$$
\begin{aligned}
& \mathscr{B} u=0 \Rightarrow q(u)=0 \Rightarrow \bar{q}_{0}(u)=0 \Rightarrow u \in D(\mathscr{C}) \text { and } \mathscr{C} u=0 \\
& \Rightarrow u \in D(\mathscr{A}) \text { and } \mathscr{A} u=0 \text {. }
\end{aligned}
$$

Now clearly $(\mathscr{B}-\mathscr{A})_{\mid D(\mathscr{A}) \cap D(\mathscr{K})}=\mathbf{K}_{\mid D(\mathscr{A}) \cap D(\mathscr{K})}$, so letting $v \in D(\mathscr{A}) \cap D(\mathscr{K})$ :

$$
(\mathscr{K} v, u)=(\mathscr{B} v, u)-(\mathscr{A} v, u)=\left(v, \mathscr{B}^{*} u\right)-\left(v, \mathscr{A}^{*} u\right)=0,
$$

so $\mathscr{K}^{*} u=-\mathscr{K} u=0$. Here we have used a well known fact about $m$-accretive operators, namely: If $G$ is $m$-accretive, $u \in D(G)$ and $G u=0$, then $u \in D\left(G^{*}\right)$ and $G^{*} u=0$. The proof of the lemma is now complete.

Now by unicity of the invariant measure $\mu$, the equation $\mathscr{A} u=0$ has only solutions of the type

$$
u=1 \otimes C \text { with } C \in \mathbf{H} \otimes \mathbf{H} .
$$

So we need only to consider the equation

$$
\mathscr{K} C=0 .
$$


Looking at $C$ as an operator on $\mathscr{H}$, this is equivalent to

$$
\left[e^{-i H(\xi) t}, C\right]=0 \text { for any } t \geqq 0 \text { and } \xi \in E \text {. }
$$

Then the spectral projections of the self-adjoint compact operator $C^{*} C$ all commute with the unitaries $e^{-i H(\xi) t}$. This means that for $\left(\mu\right.$-a.a.) $\xi_{0} \in E$, any such projection (associated with a positive eigenvalue) can be written as

$$
P=\sum_{n=1}^{N} f_{n} \otimes f_{n}
$$

where the $f_{n}$ are normalised eigenfunctions of $H\left(\xi_{0}\right)$. The invariance condition (A.1) now implies

with

$$
[V(\xi, x)-V(\xi, y)] P(x, y)=Q(x, y)
$$

$$
Q(x, y)=\left\{\left[H_{0} \otimes I-I \otimes H_{0}\right] P\right\}(x, y) .
$$

To exclude such a possibility we now formulate two conditions on $V$ :

(LB) Local boundedness: for fixed $\xi \in E$, there is a closed set $\Gamma \subset R^{v}$ of measure zero so that $R^{v} \backslash \Gamma$ is connected and so that $V(\xi,$.$) is bounded on any$ compact subset of $R^{v} \backslash \Gamma$.

(LI) Local irreducibility; there are two disjoint non-empty open sets $\mathcal{O}_{1}$, $\mathcal{O}_{2} \subset R^{v}$ such that for almost all $x \in \mathcal{O}_{1}, y \in \mathcal{O}_{2}$ :

$$
V(., x)-V(., y)
$$

is not $\mu$-a.s. constant.

These two conditions will be very easy to verify in almost all applications. Clearly (LI) implies:

$$
P(x, y)=0 \quad \text { on } \mathcal{O}_{1} \times \mathcal{O}_{2} .
$$

So for any $g \in \mathscr{H}$ supported in $\mathcal{O}_{2}$ :

$$
(P g)(x)=\sum_{n=1}^{N}\left(f_{n}, g\right) f_{n}(x)=0 \quad \text { on } \mathcal{O}_{1} .
$$

Grouping in this equation the eigenfunctions according to their eigenvalues we obtain

$$
\left\{\begin{array}{l}
\sum_{n=1}^{M} \widetilde{f}_{n}(x)=0 \quad \text { on } \mathcal{O}_{1} \\
H\left(\xi_{0}\right) \widetilde{f}_{n}=E_{n} \widetilde{f}_{n} \quad E_{1}<E_{2}<\cdots<E_{M} .
\end{array}\right.
$$

By locality of the Schrödinger operator $H\left(\xi_{0}\right)$ this further implies

$$
\left\{H\left(\xi_{0}\right)^{k} P g\right\}(x)=\sum_{n=1}^{M} E_{n}^{k} \widetilde{f}_{n}(x)=0 \text { on } \mathcal{O}_{1}, \text { for } k=0,1, \ldots, M-1 .
$$

But since

$$
\operatorname{det}\left[\begin{array}{ccccc}
1 & E_{1} & E_{1}^{2} & \cdots \cdots & E_{1}^{M-1} \\
1 & E_{2} & E_{2}^{2} & \cdots \cdots & E_{2}^{M-1} \\
\vdots & \vdots & \vdots & & \vdots \\
1 & E_{M} & E_{M}^{2} & \cdots \cdots & E_{M}^{M}-1
\end{array}\right]=\prod_{k<j}\left(E_{k}-E_{j}\right) \neq 0
$$


it follows $f_{n}(x)=0$ on $\mathcal{O}_{1}$, for $n=1, \ldots, M$.

Now by (LB), the unique continuation theorem for Schrödinger operators (see [15]) yields

$$
f_{n}=0 \text {, i.e. } P g=0 \text {. }
$$

Then $(g, P g)=\sum_{n=1}^{N}\left|\left(f_{n}, g\right)\right|^{2}=0$, i.e.:

or

$$
\left(f_{n}, g\right)=0 \text { for any } g \in \mathscr{H} \text { supported on } \mathcal{O}_{2}
$$

$$
f_{n}=0 \text { on } \mathcal{O}_{2},
$$

so that a new application of the unique continuation theorem gives $f_{n}=0$, which contradicts the hypothesis $\left\|f_{n}\right\|=1$.

We have thus proven the

Lemma. Under the assumptions (A), (B), (C) and:

(i) $A$ is m-sectorial,

(ii) $(L B)$,

(iii) $(L I)$,

the condition $(\Sigma)$ is satisfied.

Acknowledgement. I would like to thank Jürg Fröhlich and Walter Hunziker for constant interest in this work, and also Daniel Paquet for many interesting discussions.

\section{References}

1. Anderson, P. W.: Absence of diffusion in certain random lattices. Phys. Rev. 109, 1492 (1958)

2. Fröhlich, J., Spencer, T.: Absence of diffusion in the Anderson tight binding model for large disorder or low energy, Commun. Math. Phys. 88, 151-184, (1983); Mathematical theory of Anderson localization. Physica 124A, 303-310 (1984)

3. Kunz, H., Souillard, B.: Sur le spectre des operateurs aux différences finies aléatoires, Commun. Math. Phys. 78, 201-246 (1980)

4. Ovchinnikov, A. A., Erikhman, H. S.: Motion of a quantum particle in a stochastic medium. Sov. Phys. JETP 40, 733-737 (1975); Madhukar, A., Post, W.: Exact solution for the diffusion of a particle in a medium with site diagonal and off-diagonal dynamic disorder. Phys. Rev. Lett. 39 (1977) 14241427

5. Beck, A., Schwartz, J. T.: Proc. Am. Math. Soc. 8, 1049-1059 (1957); A. Beck in: Ergodic theory. F. B. Wright (ed.). London: Academic Press 1968

6. Yosida, K.: Functional analysis. Berlin-Göttingen-Heidelberg Springer 1965

7. Davies, E. B.: One parameter semigroups. London: Academic Press 1980

8. Reed, M., Simon, B.: Fourier analysis, self adjointness. New York: Academic Press 1979

9. Reed, M., Simon, B.: Scattering theory. New York: Academic Press, 1979

10. Kato, T.: J. Fac. Sci. Univ. Tokyo, Sect. IA Math. 17, 241-258 (1970)

11. Enss, V., Veselić, K.: Bound states and propagating states for time-dependent Hamiltonians. Ann. Inst. Poincaré A39, 159 (1983)

12. Ruelle, D.: Nuovo Cimento 59A, 655-662 (1969)

13. Amrein, W. O., Georgescu, V.: On the characterization of bound states and scattering states in quantum mechanics. Helv. Phys. Acta 46, 635-657 (1973)

14. Kato, T.: Perturbation theory for linear operators. Berlin, Heidelberg, New York: Springer 1984

15. Reed, M., Simon, B.: Analysis of operators. New York: Academic Press 1978 
16. Paquet, D., Leroux-Hugon P.: Electron propagation in a Markovian time-fluctuating medium: A dynamical generalization of the coherent-potential approximation. Phys. Rev. B29, 593-608 (1984)

17. Jafaev, D. R.: Sovi. Math. Dokl. 21, 545-549 (1980)

18. Jafaev, D. R.: Funct. Anal. Appl. 14, 325-326 (1980)

19. Davies, E.: Math. Ann. 210, 149 (1974)

20. Howland, J.: Math. Ann. 207, 315 (1974)

21. Yajima, K.: Resonances for the AC-stark effect. Commun. Math. Phys. 87, 331-352 (1982)

22. Yajima, K., Kitada, N.: Bound states and scattering states for time periodic Hamiltonians. Ann. Inst. Henri Poincaré A39, 145-157 (1985)

23. Bellissard, J.: In: Chaotic behaviour in quantum systems, G. Casati (ed). New York: Plenum Press, 1984

24. Bellissard, J.: Stability and instability in quantum mechanics. Preprint 1984

Communicated by T. Spencer

Received February 12, 1985; in revised form May 29, 1985 